in vivo $32: 611-623(2018)$

doi:10.21873/invivo.11283

\title{
Meta-analysis of Robot-assisted Versus Laparoscopic Surgery for Rectal Cancer
}

\author{
HIROSHI OHTANI ${ }^{1}$, KIYOSHI MAEDA $^{2}$, SHINYA NOMURA ${ }^{1}$, OSAMU SHINTO ${ }^{1}$, \\ YOKO MIZUYAMA ${ }^{1}$, HIROJI NAKAGAWA ${ }^{1}$, HISASHI NAGAHARA ${ }^{2}$, MASATSUNE SHIBUTANI $^{2}$, \\ TATSUNARI FUKUOKA ${ }^{2}$, RYOSUKE AMANO ${ }^{2}$, KOSEI HIRAKAWA ${ }^{2}$ and MASAICHI OHIRA ${ }^{2}$ \\ ${ }^{1}$ Department of Surgery, Ohno Memorial Hospital, Osaka, Japan; \\ ${ }^{2}$ Department of Surgical Oncology, Osaka City University Graduate School of Medicine, Osaka, Japan
}

\begin{abstract}
Background/Aim: A meta-analysis was conducted to evaluate and compare the short- and long-term outcomes of robot-assisted (RAS) and conventional laparoscopic surgery (LAS) for rectal cancer. Materials and Methods: We searched MEDLINE for relevant papers published between 2010 and December 2017 by using specific search terms. We analyzed outcomes over short- and long-term periods. Results: We identified 23 papers reporting results that compared RAS for rectal cancer with LAS. Our metaanalysis included 4,348 patients with rectal cancer; 2,068 had undergone RAS, and 2,280 had undergone LAS. In the short- and long-term period, 27 and 7 outcome variables were examined, respectively. RAS for rectal cancer was significantly associated with a greater operative time and a lower conversion rate to open surgery in the short-term, and results in almost similar outcomes in the long-term, compared to LAS. Conclusion: RAS may be an acceptable surgical treatment option compared to LAS for rectal cancer.
\end{abstract}

Laparoscopy-assisted surgery (LAS) for colorectal cancer (CRC) was first described in 1991 (1) and has since been widely applied by surgeons to treat patients with CRC. Several randomized trials and meta-analyses described that LAS for colon cancer results in smaller surgical incisions, reducing intraoperative blood loss, shorter recovery and hospital stays, and similarity of morbidity rate in the short-

This article is freely accessible online.

Correspondence to: Hiroshi Ohtani, Department of Surgery, Ohno Memorial Hospital, 1-26-10, Minamihorie, Nishi-ku, Osaka 5500015, Japan. Tel: +81 665311815, Fax: +81 665366390, e-mail: m5051923@msic.med.osaka-cu.ac.jp

Key Words: Rectal cancer, robot-assisted surgery, laparoscopic surgery, meta-analysis. term, and is oncologically equivalent in the long-term, compared to open surgery (OS) $(2,3)$. The application of LAS for rectal cancer was controversial in the previous Medical Research Council Conventional versus Laparoscopic-Assisted Surgery In Colorectal Cancer (MRC CLASICC) trial (4). In 2013, the MRC CLASICC indicated that long-term results continue to support the use of LAS for both colonic and rectal cancer (5). In several randomized trials and our meta-analysis, LAS for rectal cancer is described to have the benefits of reducing intraoperative blood loss, earlier resumption of oral intake, and shorter duration of hospital stay in the short-term, and equivalent long-term outcomes, with comparison to OS $(6,7)$. However, LAS for rectal cancer has technical disadvantages such as inadequate two-dimensional view with a movable video camera, a limited range for maneuver of the long, straight and rigid laparoscopic instruments in the narrow pelvic cavity, and a reduction in tactile sense.

Robot-assisted surgery (RAS) for rectal cancer was introduced to compensate for these disadvantages of LAS. Several studies describe safety and feasibility of RAS for rectal cancer after RAS for CRC was first reported in 2002 (8). The advantages of RAS are a stable 3-dimensional view, an increased dexterity for maneuvering instruments with excellent ergonomics, and physiologic tremor filtering. RAS for rectal cancer may be of use to manipulate instruments in the narrow pelvic cavity.

The value of RAS for rectal cancer has remained controversial because the short- and long-term outcomes have not been clarified. To accurately evaluate the efficacy of RAS for rectal cancer, the short- and long-term outcomes of RAS for rectal cancer must be compared to those of LAS. However, there were few randomized controlled trials comparing RAS with LAS for rectal cancer. The methodological index for non-randomized studies (MINORS) is a valid instrument for assessing the methodological quality of non-randomized studies, especially for the purposes of 
meta-analysis (9). Here, we conducted a meta-analysis of previously conducted non-randomized controlled trials (1032). The outcomes of each of the surgical procedures were analyzed at short- and long-term periods.

\section{Materials and Methods}

Study designs. There were few randomized controlled trials comparing RAS versus LAS for rectal cancer. Non-randomized studies that compared the short- and long-term outcomes of RAS versus LAS for rectal cancer were considered for this meta-analysis.

Literature search. To identify papers relevant to our study, we searched the major medical databases - MEDLINE, EMBASE, Science Citation Index, and the Cochrane Controlled Trial Register-for studies published between 2010 and December 2017. The following search terms were used: "robotic", "robot-assisted", "laparoscopic", "laparoscopy-assisted", "surgery", "rectal cancer", and "colorectal cancer". Appropriate data from the studies were used for this meta-analysis. This meta-analysis was prepared in accordance with the Preferred Reporting Items for Systemic reviews and Meta-Analysis (PRISMA) statement (Figure 1) (33).

Inclusion criteria. To enter this meta-analysis, studies had to: [1] be written in English; [2] compare RAS with LAS for rectal cancer; and [3] report on at least one of the outcome measures mentioned below.

Exclusion criteria. Studies were excluded from this analysis if the outcomes of interest were not reported for the two surgical techniques.

Study quality. The MINORS was used to evaluate the methodological quality of the non-randomized studies. Two reviewers independently appraised the studies. Disagreements were resolved by discussion and consensus.

Data extraction. Three researchers (H.O., S.N., and H.N.) extracted data from each article by using a structured sheet and entered the data into a database. We conducted meta-analyses for short- and long-term. For the short-term analysis, we collected data on the perioperative period, clinical course, postoperative complications, and pathological findings. For the perioperative period, we collected data on operation time, estimated blood loss, intraoperative transfusion, conversion rate to open surgery, perioperative mortality, the rate of the patients with lower rectal cancer and undergoing the neoadjuvant radiochemotherapy, and cost of surgery. Time to bowel movement and oral diet, duration of hospital stay were examined as the data of clinical course. For the postoperative complications, overall complications, postoperative bleeding, anastomotic leakage, interperitoneal abscess, wound infection, ileus, and deep venous thrombosis (DVT) were analyzed. Number of retrieved lymph nodes, length of circumferential resection margin (CRM), proximal margin (PM) and distal margin (DM), number of cases with inadequate CRM, DM and TME, tumor size of the specimen, and the distance from anal verge to the lower margin of tumor, were examined for the pathological data. For the long-term analysis, we used data on the rate of overall recurrence, local recurrence, distant recurrence, 3-

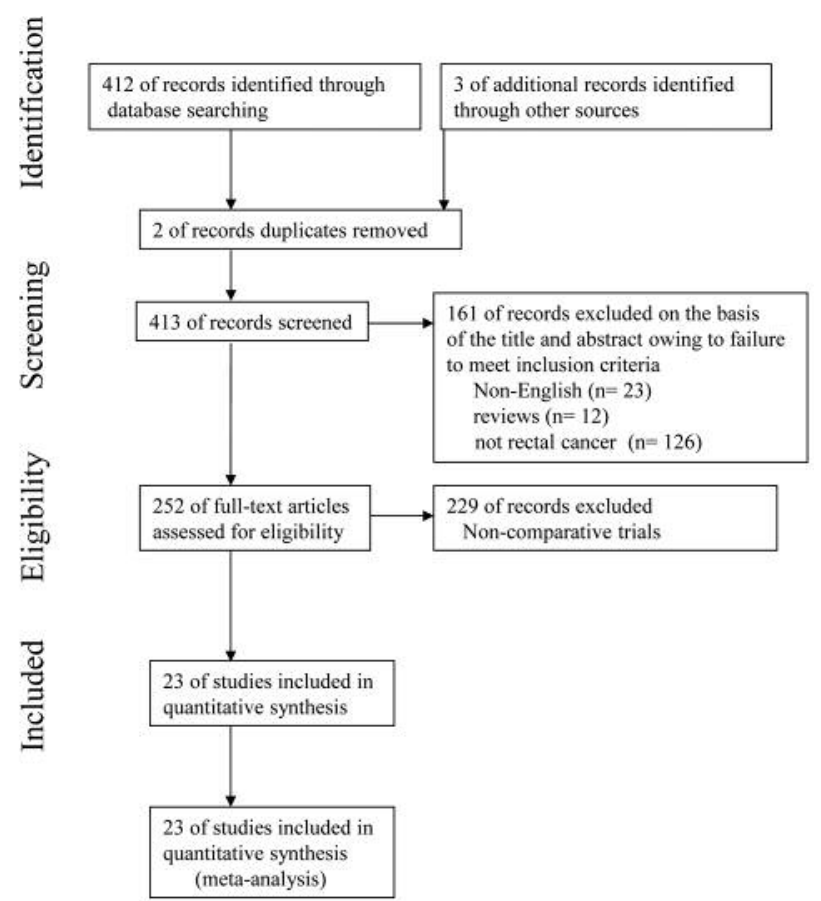

Figure 1. Flow diagram of this meta-analysis in accordance with PRISMA Statement.

year overall mortality and disease-free survival, urinary retention and erectile dysfunction.

Statistical analysis. Weighted mean differences (WMDs) and odds ratios were used for the analysis of continuous and dichotomous variables, respectively. Random effects models were used to identify heterogeneity between the studies, and the degree of heterogeneity was assessed using the $\chi^{2}$ test. For the analysis of the conversion rate, the $\chi^{2}$ test was used. The confidence interval (CI) was established at $95 \%$, and $p$-values of less than 0.05 were considered to indicate statistical significance. For the computation of the CI, estimates of the mean and standard deviation were obtained using formulas proposed by Hozo et al. (34). Statistical analyses were performed using the Review Manager (RevMan) software, version 5.3, provided by the Cochrane Collaboration, Copenhagen, Denmark.

\section{Results}

Search results. The present meta-analysis met the PRISMA statement. Overall, 412 citations were retrieved from the search strategy. Three additional articles were identified by contacting clinical experts and searching bibliographies. Two studies were excluded because of duplicate reports. One hundred and sixty-one studies were removed from the 413 because they were not written in English, reported carcinomas of the other organs except the rectum, and were described in the form of review. Two hundred and twenty-nine studies were excluded on account of non-comparative trials. 
We identified 23 trials that compared RAS with LAS for rectal cancer for this meta-analysis. The characteristics of each trial are presented in Table I. Our meta-analysis included 4348 patients with rectal cancer; of these, 2068 had undergone RAS, and 2280 had undergone LAS. Short- and long-term results are shown in Figure 2 and Figure 3, respectively. The study quality by using the MINORS was shown in Table II.

Short-term outcomes. The operative time for RAS was significantly greater, by $44.8 \mathrm{~min}$, than that for LAS (weighed mean difference $=44.80 ; 95 \% \mathrm{CI}=28.44-61.15$; $p<0.00001)$. No significant difference was found in the intraoperative estimated blood loss (Figure 2a), number of patients receiving intraoperative transfusion, the time to bowel movement, the time to oral diet, and the duration of hospital stay between RAS and LAS from the analysis. The occurrence rate of overall postoperative complications, postoperative bleeding, anastomotic leakage, interperitoneal abscess, wound infection, ileus, and DVT did not differ significantly between the two procedures (Figure 2b). Examining 3232 resections (1548 RAS and 1684 LAS), 0 and 3 perioperative mortality occurred among patients who underwent RAS and LAS, respectively. The cause of death in the 3 cases of LAS was 2, cardiovascular event, remaining 1 case was unknown.

No significant difference was found in number of retrieved lymph nodes, length of CRM, DM and PM, number of cases with inadequate CRM and DM, and number of patients with incomplete TME between RAS and LAS.

The rate of the patients undergoing the neoajuvant chemoradiotherapy and with lower rectal cancer was significantly higher in RAS than in LAS. The distance from anal verge to the lower margin of tumor was significantly shorter in RAS than in LAS (Figure 2c). No significant difference was found in the tumor size of the specimen between the 2 groups.

Conversion rate. The conversion rate from RAS to OS, and LAS to OS ranged from 0 to $9.1 \%$, and 0 to $32 \%$ in the analysis of 23 studies (Table I). The conversion rate of RAS was significantly lower than that of LAS.

Cost of surgery. In an analysis of the cost of surgery, the cost of RAS was 1.26-1.61 times higher than that of LAS.

\section{Long-term outcomes}

Tumor recurrence. The rate of overall recurrence, metastatic recurrence, and local recurrence were not significant different between RAS and LAS (Figure 3a), from the analysis of 1932 resections (953 RAS and 979 LAS), 2261 resections (1130 RAS and 1131 LAS) and 2016 resections (1000 RAS and 1016 LAS), respectively.
Long-term mortality. We found no significant difference in 3-year overall survival rate and 3-year disease free survival rate between RAS and LAS in the analysis of 1241 resections (654 RAS and 587 LAS; Figure 3a) and 1193 resections (654 RAS and 539 LAS), respectively.

Long-term morbidity. Nine and 4 articles reported data on urinary and sexual dysfunction, respectively (Figure 3b).

Urinary dysfunction did not differ significantly between the 2 groups (Odds Ratio $=0.85 ; 95 \% \mathrm{CI}=057-1.26 ; p=0.41$ ). There was no significant difference in erectile dysfunction between robotic and laparoscopic groups (Odds Ratio $=0.54$; $95 \% \mathrm{CI}=0.19-1.58 ; p=0.26)$.

Heterogeneity. In the short-term period, significant heterogeneity was found between studies with respect to operative time, estimated blood loss, rate of the patients with lower rectal cancer and undergoing the neoadjuvant radiography, cost of surgery, time to bowel movement, time to oral diet, duration of hospital stay, number of retrieved lymphnodes, length of PM and DM, the distance from anal verge to the lower margin of tumor. There was no significant heterogeneity in number of the patients receiving intraoperative transfusion, conversion rate to open surgery, perioperative mortality, overall complications, postoperative bleeding, anastomotic leakage, interperitoneal abscess, wound infection, ileus, DVT, length of CRM, number of cases with inadequate CRM, DM and TME, and tumor size of the specimen. In the long-term period, we found no significant heterogeneity in all the types of recurrence, survival rate, and rate of dysfunction between studies.

\section{Discussion}

The short- and long-term outcomes of RAS and LAS for rectal cancer were compared in this meta-analysis. While there have been few randomized trials comparing RAS to LAS for rectal cancer, several non-randomized trials have been reported (10-32).

In the short-term period, this analysis of pooled data revealed that RAS for rectal cancer was associated with a significantly longer operative time, by $44.8 \mathrm{~min}$. Although most surgeons are suspected to be already skilled to LAS, they may be in learning curve because RAS is a new procedure. Another reason may be that the set-up time for RAS is longer than that of LAS. Significant heterogeneity of the operation time between studies may be depend on the difference of points on learning curve of the surgeons, surgical procedures, tumor condition, and the factors of patients which are obesity, adhesion, and so on. The learning curve of LAS and RAS for colorectal cancer is described to be from 30 to 70 cases, and from 20 to 40 cases, respectively (29). The operation time for RAS will be expected to decrease in future, as surgeons experience RAS. 
Table I. Characteristics of all the trials.

\begin{tabular}{|c|c|c|c|c|c|c|c|c|c|c|c|c|c|c|c|c|}
\hline & \multirow[t]{2}{*}{ Year } & \multirow[t]{2}{*}{$\begin{array}{l}\text { Ref. } \\
\text { no. }\end{array}$} & \multirow[t]{2}{*}{$\begin{array}{c}\text { Style } \\
\text { of } \\
\text { study }\end{array}$} & \multicolumn{3}{|c|}{$\begin{array}{c}\text { Number } \\
\text { of } \\
\text { patients }\end{array}$} & \multicolumn{2}{|c|}{$\begin{array}{c}\text { Conversion } \\
\text { to OS } \\
\text { (or HALS) }\end{array}$} & $\begin{array}{l}\text { Reasons } \\
\text { for } \\
\text { conversion }\end{array}$ & \multicolumn{2}{|c|}{$\begin{array}{l}\text { Operation } \\
\text { method }\end{array}$} & \multicolumn{2}{|c|}{$\begin{array}{c}\text { Preoperative } \\
\text { treatment }\end{array}$} & \multicolumn{3}{|c|}{$\begin{array}{l}\text { Follow-up } \\
\text { period }\end{array}$} \\
\hline & & & & RAS & LAS & RAS & LAS & RAS & LAS & RAS & LAS & RAS & LAS & entire & RAS & LAS \\
\hline $\begin{array}{l}1 \text { Bianchi } \\
\text { PP et al. }\end{array}$ & 2010 & 10 & $\begin{array}{c}\text { pro- } \\
\text { spective }\end{array}$ & $e^{25}$ & 25 & $\begin{array}{c}0 \\
(0 \%)\end{array}$ & $\begin{array}{c}1 \\
(4 \%)\end{array}$ & - & $\begin{array}{l}\text { ischemia } \\
\text { of the } \\
\text { left colon }\end{array}$ & $\begin{array}{c}\mathrm{AR} \\
(72 \%)\end{array}$ & $\begin{array}{c}\mathrm{AR} \\
(76 \%)\end{array}$ & $\begin{array}{c}\text { CRT } \\
(52 \%) \\
\text { APR } \\
(28 \%)\end{array}$ & $\begin{array}{c}\text { CRT } \\
(40 \%) \\
\text { APR } \\
(24 \%)\end{array}$ & $\begin{array}{c}10 \mathrm{M} \\
\text { (mean) }\end{array}$ & $\mathrm{U}$ & $\mathrm{U}$ \\
\hline $\begin{array}{l}2 \text { Popescu } \\
\text { et al. }\end{array}$ & 2010 & 11 & $\begin{array}{l}\text { retro- } \\
\text { spective }\end{array}$ & $e^{38}$ & 84 & $\begin{array}{c}2 \\
(5.2 \%)\end{array}$ & $\begin{array}{c}9 \\
(10.5 \%)\end{array}$ & $\begin{array}{l}\text { Adhesion, } \\
\text { bleeding } \\
\text { tumor } \\
\text { condition }\end{array}$ & & $\begin{array}{c}\text { AR } \\
(79 \%) \\
\text { APR } \\
(21 \%)\end{array}$ & $\begin{array}{c}\text { AR } \\
(60 \%) \\
\text { APR } \\
(40 \%)\end{array}$ & $\mathrm{U}$ & $\mathrm{U}$ & $\mathrm{U}_{(\mathrm{r}}$ & $\begin{array}{c}13 \mathrm{M} \\
\text { (median) }\end{array}$ & $\begin{array}{c}27.5 \mathrm{M} \\
\text { (median) }\end{array}$ \\
\hline $\begin{array}{l}3 \text { Park JS } \\
\text { et al. }\end{array}$ & 2011 & 12 & $\begin{array}{c}\text { pro- } \\
\text { spective }\end{array}$ & $e^{52}$ & 123 & $\begin{array}{c}0 \\
(0 \%)\end{array}$ & $\begin{array}{c}0 \\
(0 \%)\end{array}$ & - & - & $\begin{array}{c}\text { AR } \\
\text { APR }\end{array}$ & $\begin{array}{c}\text { AR } \\
\text { APR }\end{array}$ & $\begin{array}{c}\text { CRT } \\
(23.1 \%)\end{array}$ & $\begin{array}{c}\text { CRT } \\
(10.2 \%)\end{array}$ & $\mathrm{U}$ & $\mathrm{U}$ & $\mathrm{U}$ \\
\hline $\begin{array}{l}4 \mathrm{Kwak} \\
\text { JM et al. }\end{array}$ & 2011 & 13 & $\begin{array}{l}\text { retro- } \\
\text { spective }\end{array}$ & $e^{59}$ & 59 & $\begin{array}{c}0 \\
(0 \%)\end{array}$ & $\begin{array}{c}2 \\
(3.4 \%)\end{array}$ & - & $\begin{array}{l}\text { Difficulty } \\
\text { in pelvic } \\
\text { dissection }\end{array}$ & $\begin{array}{c}\mathrm{AR} \\
(91.5 \%) \\
\mathrm{ISR} \\
(8.5 \%) \\
\text { APR } \\
(0 \%)\end{array}$ & $\begin{array}{c}\text { AR } \\
(88.1 \%) \\
\text { ISR } \\
(10.2 \%) \\
\text { APR } \\
(1.7 \%)\end{array}$ & $\begin{array}{l}\text { CRT } \\
(13.6 \%)\end{array}$ & $\begin{array}{c}\text { CRT } \\
(8.5 \%)\end{array}$ & $\mathrm{U}_{(\mathrm{r}}$ & $\begin{array}{c}17 \mathrm{M} \\
\text { (median) }\end{array}$ & $\begin{array}{c}13 \mathrm{M} \\
\text { (median) }\end{array}$ \\
\hline $\begin{array}{l}5 \text { Baek } \\
\text { SJ et al. } \\
*\end{array}$ & 2012 & 14 & $\begin{array}{l}\text { retro- } \\
\text { spective }\end{array}$ & $e^{154}$ & 150 & $\mathrm{U}$ & $\mathrm{U}$ & $\mathrm{U}$ & $\mathrm{U}$ & $\begin{array}{c}\text { AR } \\
(69.4 \%) \\
\text { ISR } \\
(23.4 \%) \\
\text { APR } \\
(7.1 \%)\end{array}$ & $\begin{array}{c}\text { AR } \\
(86.7 \%) \\
\text { ISR } \\
(9.3 \%) \\
\text { APR } \\
(2.7 \%) \\
\text { IPAA } \\
(1.3 \%)\end{array}$ & $\begin{array}{c}\text { CRT } \\
(22.7 \%)\end{array}$ & $\begin{array}{l}\text { CRT } \\
(8 \%)\end{array}$ & $\mathrm{U}$ & $\mathrm{U}$ & $\mathrm{U}$ \\
\hline $\begin{array}{l}6 \text { Saklani } \\
\text { AP et al. }\end{array}$ & 2013 & 15 & $\begin{array}{l}\text { retro- } \\
\text { spective }\end{array}$ & $e^{74}$ & 64 & $\begin{array}{c}1 \\
(1.4 \%)\end{array}$ & $\begin{array}{c}4 \\
(6.3 \%)\end{array}$ & $\begin{array}{c}\text { Early } \\
\text { experience }\end{array}$ & $\begin{array}{c}\text { Early } \\
\text { experience }\end{array}$ & $\begin{array}{c}\text { AR } \\
(62.2 \%) \\
\text { ISR } \\
(4.1 \%) \\
\text { CAA } \\
(31.1 \%) \\
\text { APR } \\
(2.7 \%)\end{array}$ & $\begin{array}{c}\text { AR } \\
(71.9 \%) \\
\text { ISR } \\
(1.6 \%) \\
\text { CAA } \\
(21.9 \%) \\
\text { APR } \\
(4.7 \%)\end{array}$ & $\mathrm{U}$ & $\mathrm{U}$ & $\begin{array}{c}30.1 \pm \\
9.9 \mathrm{M} \\
\text { (mean) }\end{array}$ & U & $\mathrm{U}$ \\
\hline $\begin{array}{l}7 \text { Baek SJ } \\
\text { et al. } \\
* *\end{array}$ & 2013 & 16 & $\begin{array}{l}\text { retro- } \\
\text { spective }\end{array}$ & 47 & 37 & $\begin{array}{c}1 \\
(2.1 \%)\end{array}$ & $\begin{array}{c}6 \\
(16.2 \%)\end{array}$ & $\mathrm{U}$ & $\mathrm{U}$ & $\begin{array}{c}\mathrm{AR} \\
(87.2 \%) \\
\mathrm{ISR} \\
(12.8 \%)\end{array}$ & $\begin{array}{c}\mathrm{AR} \\
(89.2 \%) \\
\mathrm{ISR} \\
(10.8 \%)\end{array}$ & $\begin{array}{c}\text { CRT } \\
(42.6 \%)\end{array}$ & $\begin{array}{c}\text { CRT } \\
(32.4 \%)\end{array}$ & $\begin{array}{c}31.5 \mathrm{M} \\
(\text { median })\end{array}$ & I U & $\mathrm{U}$ \\
\hline $\begin{array}{l}8 \text { Kang } \mathrm{J} \\
\text { et al. }\end{array}$ & 2013 & 17 & $\begin{array}{c}\text { pro- } \\
\text { spective }\end{array}$ & 165 & 165 & $\begin{array}{c}1 \\
(0.6 \%)\end{array}$ & $\begin{array}{c}3 \\
(1.8 \%)\end{array}$ & $\mathrm{U}$ & $\mathrm{U}$ & $\begin{array}{c}\text { SPP } \\
(99.4 \%) \\
\text { Hartmann } \\
(0.6 \%) \\
\text { APR } \\
(0 \%)\end{array}$ & $\begin{array}{c}\text { SPP } \\
(95.8 \%) \\
\text { Hartmann } \\
(0.6 \%) \\
\text { APR } \\
(3.6 \%)\end{array}$ & $\begin{array}{c}\text { CRT } \\
(23.6 \%)\end{array}$ & $\begin{array}{c}\text { CRT } \\
(21.8 \%)\end{array}$ & $\begin{array}{c}22.4 \mathrm{M} \\
(\text { median })\end{array}$ & I) $U$ & $\mathrm{U}$ \\
\hline $\begin{array}{l}9 \text { Fernandez } \\
\text { et al. }\end{array}$ & 2013 & 18 & $\begin{array}{l}\text { retro- } \\
\text { spective }\end{array}$ & $e^{13}$ & 59 & $\begin{array}{c}1 \\
(8 \%)\end{array}$ & $\begin{array}{c}10 \\
(17 \%)\end{array}$ & $\mathrm{U}$ & $\mathrm{U}$ & $\begin{array}{c}\text { AR } \\
(39 \%) \\
\text { APR } \\
(62 \%)\end{array}$ & $\begin{array}{c}\text { AR } \\
(75 \%) \\
\text { APR } \\
(25 \%)\end{array}$ & $\begin{array}{l}\text { CRT } \\
(77 \%)\end{array}$ & $\begin{array}{l}\text { CRT } \\
(54 \%)\end{array}$ & $\mathrm{U}$ & $\mathrm{U}$ & $\mathrm{U}$ \\
\hline $\begin{array}{l}10 \text { D'Annibale } \\
\text { A et al. }\end{array}$ & 2013 & 19 & $\begin{array}{l}\text { retro- } \\
\text { spective }\end{array}$ & 50 & 50 & $\begin{array}{c}0 \\
(0 \%)\end{array}$ & $\begin{array}{c}6 \\
(12 \%)\end{array}$ & - & $\mathrm{U}$ & $\begin{array}{c}\mathrm{AR} \\
(100 \%)\end{array}$ & $\begin{array}{c}\mathrm{AR} \\
(100 \%)\end{array}$ & $\begin{array}{l}\text { CRT } \\
(68 \%)\end{array}$ & $\begin{array}{l}\text { CRT } \\
(56 \%)\end{array}$ & $\mathrm{U}$ & $\mathrm{U}$ & $\mathrm{U}$ \\
\hline $\begin{array}{l}11 \text { Park SY } \\
\text { et al. }\end{array}$ & 2013 & 20 & $\begin{array}{l}\text { retro- } \\
\text { spective }\end{array}$ & 40 & 40 & $\begin{array}{c}0 \\
(0 \%)\end{array}$ & $\begin{array}{c}0 \\
(0 \%)\end{array}$ & - & - & $\begin{array}{c}\text { ISR } \\
(100 \%)\end{array}$ & $\begin{array}{c}\text { ISR } \\
(100 \%)\end{array}$ & $\begin{array}{l}\text { CRT } \\
(80 \%)\end{array}$ & $\begin{array}{l}\text { CRT } \\
(50 \%)\end{array}$ & $\mathrm{U}$ & $\mathrm{U}$ & $\mathrm{U}$ \\
\hline $\begin{array}{l}12 \mathrm{Kim} Y S \\
\text { et al. }\end{array}$ & 2016 & 21 & $\begin{array}{l}\text { retro- } \\
\text { spective }\end{array}$ & 33 & 66 & $\begin{array}{c}2 \\
(6.1 \%)\end{array}$ & $\begin{array}{c}0 \\
(0 \%)\end{array}$ & $\begin{array}{l}\text { Invasion } \\
\text { to ureter } \\
\text { ischemia } \\
\text { of the } \\
\text { left colon }\end{array}$ & - & $\begin{array}{c}\text { AR } \\
(93.9 \%) \\
\text { Hartmann } \\
(0 \%) \\
\text { APR } \\
(6.1 \%)\end{array}$ & $\begin{array}{c}\text { AR } \\
(92.4 \%) \\
\text { Hartmann } \\
(1.5 \%) \\
\text { APR } \\
(6.1 \%)\end{array}$ & $\begin{array}{c}\text { CRT } \\
(100 \%)\end{array}$ & $\begin{array}{c}\text { CRT } \\
(100 \%)\end{array}$ & $\mathrm{U}$ & $\mathrm{U}$ & $\mathrm{U}$ \\
\hline
\end{tabular}




\begin{tabular}{|c|c|c|c|c|c|c|c|c|c|c|c|c|c|c|c|c|}
\hline & \multirow[t]{2}{*}{ Year } & \multirow[t]{2}{*}{$\begin{array}{l}\text { Ref. } \\
\text { no. }\end{array}$} & \multirow[t]{2}{*}{$\begin{array}{l}\text { Style } \\
\text { of } \\
\text { study }\end{array}$} & \multicolumn{3}{|c|}{$\begin{array}{c}\text { Number } \\
\text { of } \\
\text { patients }\end{array}$} & \multicolumn{2}{|c|}{$\begin{array}{l}\text { Conversion } \\
\text { to OS } \\
\text { (or HALS) }\end{array}$} & $\begin{array}{l}\text { Reasons } \\
\text { for } \\
\text { conversion }\end{array}$ & \multicolumn{2}{|c|}{$\begin{array}{c}\text { Operation } \\
\text { method }\end{array}$} & \multicolumn{2}{|c|}{$\begin{array}{l}\text { Preoperative } \\
\text { treatment }\end{array}$} & \multicolumn{3}{|c|}{$\begin{array}{l}\text { Follow-up } \\
\text { period }\end{array}$} \\
\hline & & & & RAS & LAS & RAS & LAS & RAS & LAS & RAS & LAS & RAS & LAS & entire & RAS & LAS \\
\hline $\begin{array}{l}13 \text { Cho MS } \\
\text { et al. }\end{array}$ & 2015 & 22 & $\begin{array}{c}\text { retro- } \\
\text { spective }\end{array}$ & 278 & 278 & $\begin{array}{c}1 \\
(0.4 \%)\end{array}$ & $\begin{array}{c}2 \\
(0.7 \%)\end{array}$ & $\begin{array}{l}\text { Bowel } \\
\text { perfo- } \\
\text { ration }\end{array}$ & $\begin{array}{c}\text { Severe } \\
\text { tumor } \\
\text { adhesion } \\
\text { major } \\
\text { vessel } \\
\text { bleeding }\end{array}$ & $\begin{array}{c}\mathrm{AR} \\
(80.9 \%) \\
\mathrm{CAA} \\
(19.1 \%)\end{array}$ & $\begin{array}{c}\text { AR } \\
(84.5 \%) \\
\text { CAA } \\
(15.5 \%)\end{array}$ & $\begin{array}{c}\text { CRT } \\
(65.5 \%)\end{array}$ & $\begin{array}{c}\text { CRT } \\
(65.1 \%)\end{array}$ & $\begin{array}{l}51.8 \pm \\
15.3 \mathrm{M} \\
(\text { mean) }\end{array}$ & $\begin{array}{l}51.0 \pm \\
13.1 \mathrm{M} \\
\text { (mean) }\end{array}$ & $\begin{array}{c}51.0 \pm \\
13.1 \mathrm{M} \\
\text { (mean) }\end{array}$ \\
\hline $\begin{array}{l}14 \text { Baik SH } \\
\text { et al. }\end{array}$ & 2009 & 23 & $\begin{array}{c}\text { pro- } \\
\text { spective }\end{array}$ & 56 & 57 & $\begin{array}{c}0 \\
(0 \%)\end{array}$ & $\begin{array}{c}6 \\
(10.5 \%)\end{array}$ & - & $\begin{array}{c}\text { Severe } \\
\text { hemorrhage } \\
\text { severe } \\
\text { narrow } \\
\text { pelvic } \\
\text { cavity }\end{array}$ & $\begin{array}{c}\mathrm{AR} \\
(100 \%)\end{array}$ & $\begin{array}{c}\mathrm{AR} \\
(100 \%)\end{array}$ & $\begin{array}{l}\text { CRT } \\
(8.9 \%)\end{array}$ & $\begin{array}{c}\text { CRT } \\
(12.3 \%)\end{array}$ & $\begin{array}{c}14.3 \mathrm{M} \\
\text { (median) }\end{array}$ & U & $\mathrm{U}$ \\
\hline $\begin{array}{l}15 \text { Patriti A } \\
\text { et al. }\end{array}$ & 2009 & 24 & $\begin{array}{c}\text { pro- } \\
\text { spective }\end{array}$ & 29 & 37 & $\begin{array}{c}0 \\
(0 \%)\end{array}$ & $\begin{array}{c}7 \\
(18.9 \%)\end{array}$ & - & $\mathrm{U}$ & $\begin{array}{c}\mathrm{AR} \\
(65.5 \%) \\
\text { APR } \\
(17.2 \%) \\
\text { ISR } \\
(17.2 \%)\end{array}$ & $\begin{array}{c}\text { AR } \\
(86.4 \%) \\
\text { APR } \\
(8.1 \%) \\
\text { ISR } \\
(5.4 \%)\end{array}$ & $\begin{array}{c}\text { CRT } \\
(24.1 \%)\end{array}$ & $\begin{array}{l}\text { CRT } \\
(5.4 \%)\end{array}$ & $\mathrm{U}$ & $\begin{array}{l}29.2 \mathrm{M} \\
\text { (mean) }\end{array}$ & $\begin{array}{l}18.7 \mathrm{M} \\
\text { (mean) }\end{array}$ \\
\hline $\begin{array}{l}16 \mathrm{Kim} \mathrm{NK} \\
\text { et al. }\end{array}$ & 2010 & 25 & $\begin{array}{c}\text { retro- } \\
\text { spective }\end{array}$ & 100 & 100 & $\begin{array}{c}2 \\
(2 \%)\end{array}$ & $\begin{array}{c}3 \\
(3 \%)\end{array}$ & $\mathrm{U}$ & $\mathrm{U}$ & $\begin{array}{c}\text { Sphincter } \\
\text { preserving } \\
\text { surgery } \\
(98 \%)\end{array}$ & $\begin{array}{c}\text { Sphincter } \\
\text { preserving } \\
\text { surgery } \\
(99 \%)\end{array}$ & $\begin{array}{l}\text { CRT } \\
(14 \%)\end{array}$ & $\begin{array}{l}\text { CRT } \\
(46 \%)\end{array}$ & $\mathrm{U}$ & $\mathrm{U}$ & $\mathrm{U}$ \\
\hline $\begin{array}{l}17 \text { Baek JH } \\
\text { et al. }\end{array}$ & 2011 & 26 & $\begin{array}{c}\text { pro- } \\
\text { spective }\end{array}$ & 41 & 41 & $\begin{array}{c}3 \\
(7.3 \%)\end{array}$ & $\begin{array}{c}9 \\
(22 \%)\end{array}$ & $\begin{array}{l}\text { Obesity } \\
\text { with } \\
\text { narrow } \\
\text { pelvis } \\
\text { Adhesion }\end{array}$ & $\begin{array}{l}\text { Adhesion } \\
\text { to adjacent } \\
\text { organ } \\
\text { Instrument } \\
\text { too short } \\
\text { Obesity with } \\
\text { narrow } \\
\text { pelvis }\end{array}$ & $\begin{array}{c}\text { AR } \\
(80.5 \%) \\
\text { CAA } \\
(4.9 \%) \\
\text { APR } \\
\text { h }(14.6 \%)\end{array}$ & $\begin{array}{c}\text { AR } \\
(80.5 \%) \\
\text { CAA } \\
(4.9 \%) \\
\text { APR } \\
(14.6 \%)\end{array}$ & $\begin{array}{c}\text { CRT } \\
(80.5 \%)\end{array}$ & $\begin{array}{c}\text { CRT } \\
(43.9 \%)\end{array}$ & $\mathrm{U}$ & $\mathrm{U}$ & $\mathrm{U}$ \\
\hline $\begin{array}{l}18 \text { Ielpo B } \\
\text { et al. }\end{array}$ & 2014 & 27 & $\begin{array}{c}\text { retro- } \\
\text { spective }\end{array}$ & 56 & 87 & $\begin{array}{c}2 \\
(3.6 \%)\end{array}$ & $\begin{array}{c}10 \\
(11.5 \%)\end{array}$ & $\mathrm{U}$ & $\begin{array}{l}\text { difficult } \\
\text { mesorectal } \\
\text { dissection } \\
\text { border } \\
\text { tumoral } \\
\text { invasion }\end{array}$ & $\begin{array}{c}\text { AR } \\
(71.4 \%) \\
\text { APR } \\
(26.7 \%) \\
\text { CAA } \\
(1.8 \%)\end{array}$ & $\begin{array}{c}\text { AR } \\
(75.8 \%) \\
\text { APR } \\
(23 \%) \\
\text { CAA } \\
(1.1 \%)\end{array}$ & $\begin{array}{c}\text { CRT } \\
(80.5 \%)\end{array}$ & $\begin{array}{c}\text { CRT } \\
(82.1 \%)\end{array}$ & $\mathrm{U}$ & $\mathrm{U}$ & $\mathrm{U}$ \\
\hline $\begin{array}{l}19 \mathrm{Kim} \mathrm{JC} \\
\text { et al. }\end{array}$ & 2016 & 28 & $\begin{array}{c}\text { retro- } \\
\text { spective }\end{array}$ & 533 & 486 & $\begin{array}{c}1 \\
(0.2 \%)\end{array}$ & $\begin{array}{c}25 \\
(5.1 \%)\end{array}$ & $\mathrm{U}$ & $\begin{array}{l}\text { Advanced } \\
\text { cancer } \\
\text { difficult } \\
\text { mobilization }\end{array}$ & $\begin{array}{c}\text { AR } \\
(95.1 \%) \\
\text { APR } \\
(4.9 \%)\end{array}$ & $\begin{array}{c}\text { AR } \\
(97.9 \%) \\
\text { APR } \\
(2.1 \%)\end{array}$ & $\begin{array}{c}\text { CRT } \\
(32.3 \%)\end{array}$ & $\begin{array}{c}\text { CRT } \\
(12.6 \%)\end{array}$ & $\mathrm{U}$ & $\mathrm{U}$ & $\mathrm{U}$ \\
\hline $\begin{array}{l}20 \text { Park EJ } \\
\text { et al. }\end{array}$ & 2014 & 29 & $\begin{array}{c}\text { retro- } \\
\text { spective }\end{array}$ & 89 & 89 & $\begin{array}{c}0 \\
(0 \%)\end{array}$ & $\begin{array}{c}5 \\
(5.6 \%)\end{array}$ & - & U & $\begin{array}{c}\mathrm{AR} \\
(100 \%)\end{array}$ & $\begin{array}{c}\text { AR } \\
(100 \%)\end{array}$ & $\mathrm{U}$ & $\mathrm{U}$ & $\mathrm{U}$ & $\mathrm{U}$ & $\mathrm{U}$ \\
\hline $\begin{array}{l}21 \text { Morelli L } \\
\text { et al. }\end{array}$ & 2016 & 30 & $\begin{array}{l}\text { retro- } \\
\text { spective }\end{array}$ & 50 & 25 & $\begin{array}{c}1 \\
(2 \%)\end{array}$ & $\begin{array}{c}8 \\
(32 \%)\end{array}$ & $\begin{array}{l}\text { Visceral } \\
\text { obesity }\end{array}$ & $\begin{array}{l}\text { Visceral } \\
\text { obesity }\end{array}$ & $\begin{array}{c}\text { AR } \\
(64 \%) \\
\text { ISR } \\
(22 \%) \\
\text { APR } \\
(14 \%)\end{array}$ & $\begin{array}{l}\text { AR } \\
(84) \\
\text { ISR } \\
(8 \%) \\
\text { APR } \\
(8 \%)\end{array}$ & $\begin{array}{l}\text { CRT } \\
(46 \%)\end{array}$ & $\begin{array}{l}\text { CRT } \\
(36 \%)\end{array}$ & $\mathrm{U}$ & $\mathrm{U}$ & $\mathrm{U}$ \\
\hline $\begin{array}{l}22 \text { Silva- } \\
\text { Velazco } \\
\text { et al. }\end{array}$ & 2016 & 31 & $\begin{array}{c}\text { retro- } \\
\text { spective }\end{array}$ & 66 & 118 & $\begin{array}{c}6 \\
(9.1 \%)\end{array}$ & $\begin{array}{c}18 \\
(15.4 \%)\end{array}$ & $\mathrm{U}$ & $\mathrm{U}$ & $\begin{array}{c}\text { AR } \\
(65.2 \%) \\
\text { APR } \\
(33.3 \%)\end{array}$ & $\begin{array}{c}\text { AR } \\
(77.1 \%) \\
\text { APR } \\
(21.2 \%)\end{array}$ & $\begin{array}{c}\text { CRT } \\
(51.5 \%)\end{array}$ & $\begin{array}{c}\text { CRT } \\
(49.2 \%)\end{array}$ & $\mathrm{U}$ & $\mathrm{U}$ & $\mathrm{U}$ \\
\hline $\begin{array}{l}23 \text { Allemann } \\
\mathrm{P}\end{array}$ & 2016 & 32 & $\begin{array}{c}\text { pro- } \\
\text { spectively }\end{array}$ & $\mathrm{y}^{20}$ & 40 & $\begin{array}{c}1 \\
(5 \%)\end{array}$ & $\begin{array}{c}8 \\
(20 \%)\end{array}$ & Obesity & Obesity & $\begin{array}{c}\text { AR } \\
(75 \%) \\
\text { ISR } \\
(15 \%) \\
\text { APR } \\
(10 \%)\end{array}$ & $\begin{array}{c}\text { AR } \\
(62.5 \%) \\
\text { ISR } \\
(22.5 \%) \\
\text { APR } \\
(15 \%)\end{array}$ & $\begin{array}{l}\text { CRT } \\
(65 \%)\end{array}$ & $\begin{array}{l}\text { CRT } \\
(60 \%)\end{array}$ & $\begin{array}{c}22.5 \pm \\
7.0 \mathrm{M} \\
(\text { mean })\end{array}$ & $\begin{array}{l}31.8 \pm \\
17.1 \mathrm{M} \\
\text { (mean) }\end{array}$ & $\mathrm{U}$ \\
\hline
\end{tabular}

-: Not stated; U: unknown; M: month; Y: year; CRT: chemoradiation; AR: anterior resection; ISR: intersphncteric resection; APR: abdominoperitoneal resection; CAA: coloanal anastomosis; IPAA: ileal pouch-anal anastomosis; SPP: sphincter-preserving procedure. *Korea University, Anam Hospital; **Yonsei University Health System. Ref.: Reference. 


\section{Operative time (minutes)}

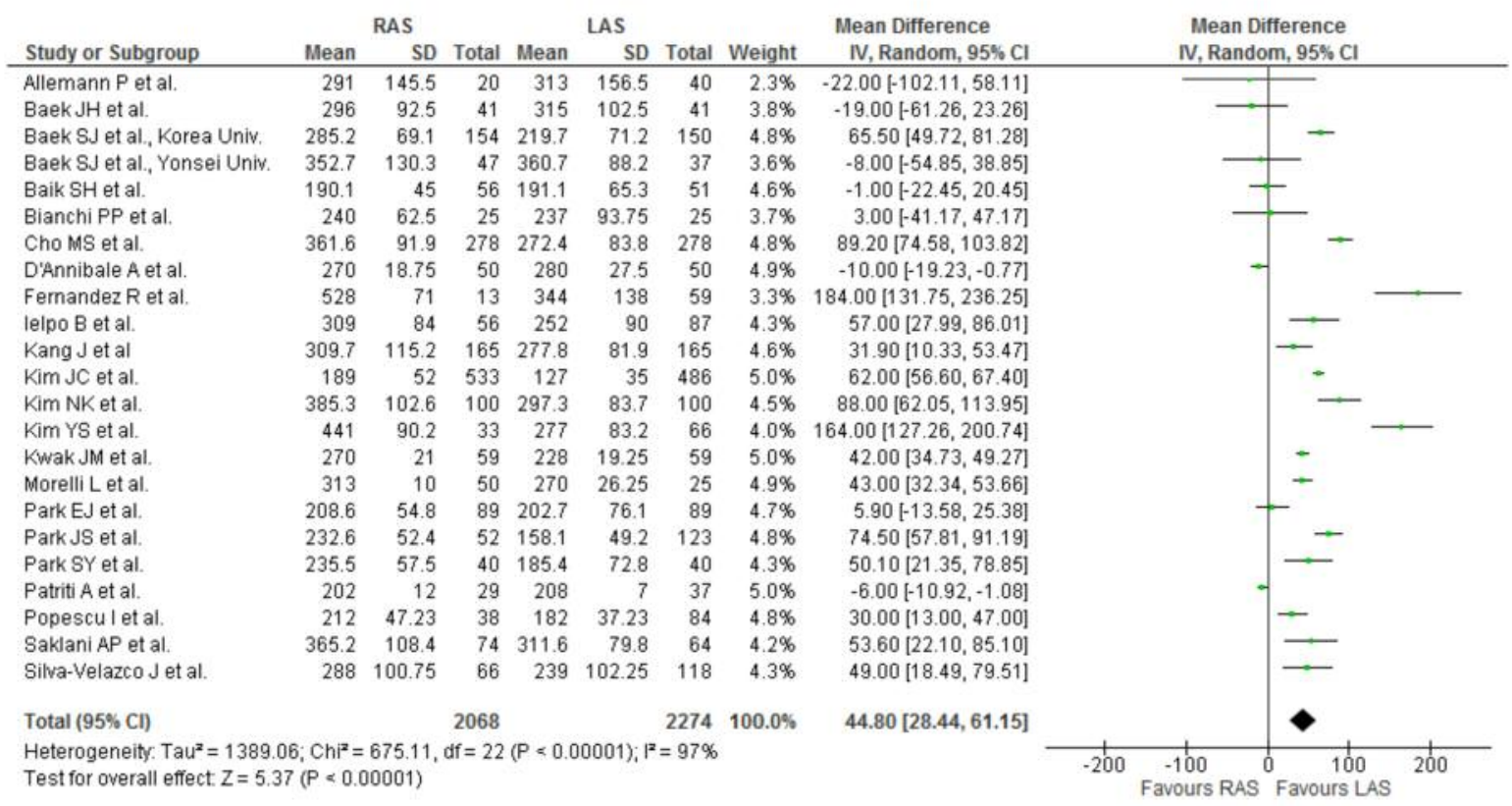

\section{Intraoperative estimated blood loss (ml)}

\begin{tabular}{|c|c|c|c|c|c|c|c|c|c|c|}
\hline Study or Subgroup & \multicolumn{3}{|c|}{ RAS } & \multicolumn{3}{|c|}{ LAS } & \multicolumn{2}{|r|}{ Mean Difference } & \multicolumn{2}{|c|}{$\begin{array}{l}\text { Mean Difference } \\
\text { IV, Random, } 95 \% \mathrm{Cl}\end{array}$} \\
\hline Allemann $\mathrm{P}$ et al. & 58 & 76 & 20 & 219 & 421 & 40 & $2.4 \%$ & $-161.00[-295.65,-26.35]$ & & \\
\hline Baek.JH et al. & 200 & 495 & 41 & 300 & 245.75 & 41 & $1.7 \%$ & $-100.00[-269.16,69.16]$ & & \\
\hline Baek SJ et al., Korea Univ. & 167.8 & 26.1 & 154 & 126.2 & 267.7 & 150 & $8.5 \%$ & $41.60[-1.44,84.64]$ & & \\
\hline Baek S.J et al., Yonsei Univ. & 190.9 & 284.7 & 47 & 302.7 & 305.3 & 37 & $2.6 \%$ & $-111.80[-239.48,15.88]$ & & \\
\hline Cho MS et al. & 179 & 236.5 & 278 & 147 & 295.3 & 278 & $8.3 \%$ & $32.00[-12.47,76.47]$ & & \\
\hline Fernandez $\mathrm{R}$ et al. & 157 & 125 & 13 & 200 & 368.75 & 59 & $3.0 \%$ & $-43.00[-159.06,73.06]$ & & \\
\hline lelpo B et al. & 280 & 35.3 & 56 & 240 & 53.7 & 87 & $11.3 \%$ & $40.00[25.41,54.59]$ & & - \\
\hline Kang $\mathrm{J}$ et al & 133 & 192.3 & 165 & 140.1 & 216.4 & 165 & $8.3 \%$ & $-7.10[-51.27,37.07]$ & & \\
\hline Kim YS et al. & 232 & 180 & 33 & 205 & 163.8 & 66 & $5.5 \%$ & $27.00[-46.03,100.03]$ & & \\
\hline Park EJ et al. & 55.8 & 119.4 & 89 & 73.2 & 157.1 & 89 & $8.7 \%$ & $-17.40[-58.40,23.60]$ & & \\
\hline Park SY et al. & 45.7 & 40 & 40 & 59.2 & 35.8 & 40 & $11.1 \%$ & $-13.50[-30.14,3.14]$ & $\rightarrow$ & \\
\hline Patriti $A$ et al. & 137.4 & 156 & 29 & 127 & 169 & 37 & $5.1 \%$ & $10.40[-68.27,89.07]$ & & \\
\hline Popescu l et al. & 100 & 50 & 38 & 150 & 50 & 84 & $10.9 \%$ & $-50.00[-69.16,-30.84]$ & $=$ & \\
\hline Saklani AP et al. & 180 & 28.1 & 74 & 210 & 35.7 & 64 & $11.5 \%$ & $-30.00[-40.84,-19.16]$ & - & \\
\hline Silva-Velazco J et al. & 235 & 741.25 & 66 & 200 & 746.25 & 118 & $1.0 \%$ & $35.00[-188.85,258.85]$ & & \\
\hline Total $(95 \% \mathrm{Cl})$ & & & 1143 & & & 1355 & $100.0 \%$ & $-9.29[-32.82,14.24]$ & & \\
\hline $\begin{array}{l}\text { Heterogeneity. Tau }{ }^{2}=1222.3 \\
\text { Test for overall effect: } Z=0.7\end{array}$ & $\begin{array}{l}\mathrm{Chi}^{2}= \\
P=0.4\end{array}$ & 94.21, & $f=14$ & $P<0.0$ & 01); & $=85 \%$ & & & $\begin{array}{cc}-200 & -100 \\
\text { Favours RAS } & 1 \\
\end{array}$ & $\begin{array}{c}100 \quad 200 \\
\text { Favours LAS }\end{array}$ \\
\hline
\end{tabular}

Figure 2. Continued

No significant difference was found in intraoperative blood loss and number of patients receiving intraoperative transfusion. The reason for significant heterogeneity of the intraoperative blood loss may be almost similar to that of the operation time.

Higher rate of the patients undergoing the neoajuvant chemoradiotherapy in the RAS group may indicate more patients with locally advanced rectal tumor in RAS, comparing to LAS. Tumor location was lower in RAS than in LAS, however, the conversion rate of RAS was significantly lower than that of LAS. The reason may be that the surgeons for RAS are able to safely accomplish surgery, using ergonomic instruments and three-dimensional view, in the narrow and enclosed pelvic cavity, while controlling intraoperative bleeding. This may contribute to the longer operation time of RAS. Choi GS et al. described that 3-dimensional images and free articulation of EndoWrist instruments allow pinpoint bleeding control and make performing intracorporeal suture easy (35). We previously 
b

Overall complications

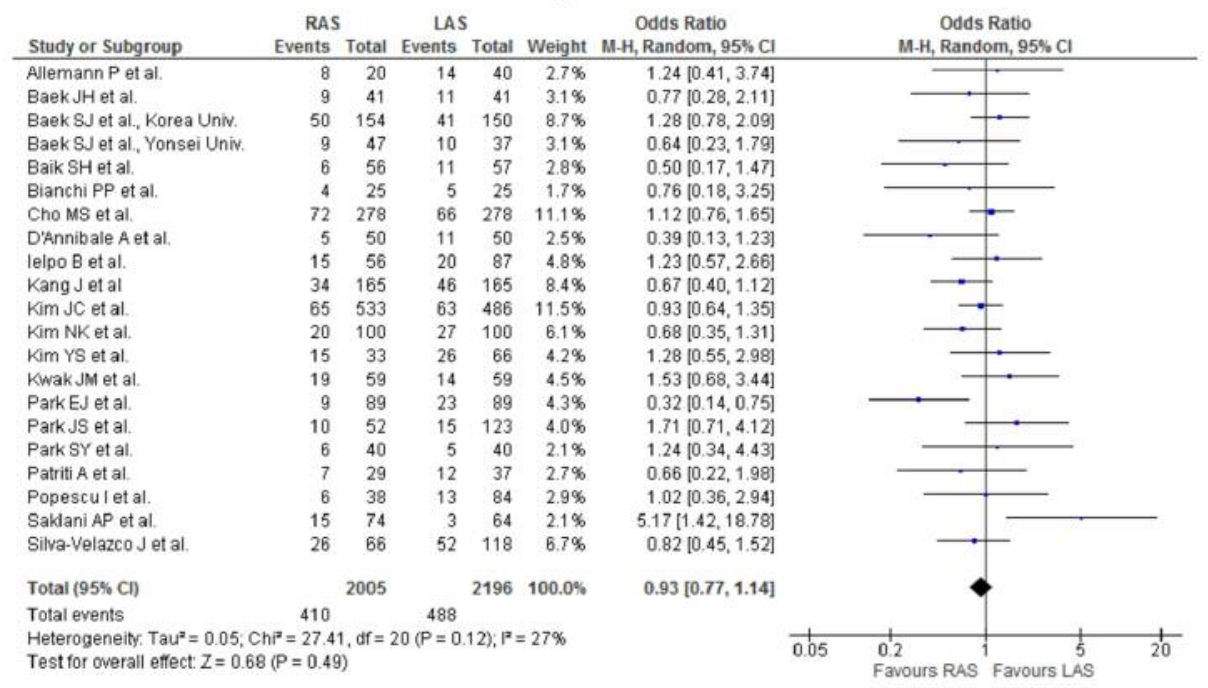

Anastomotic leakage

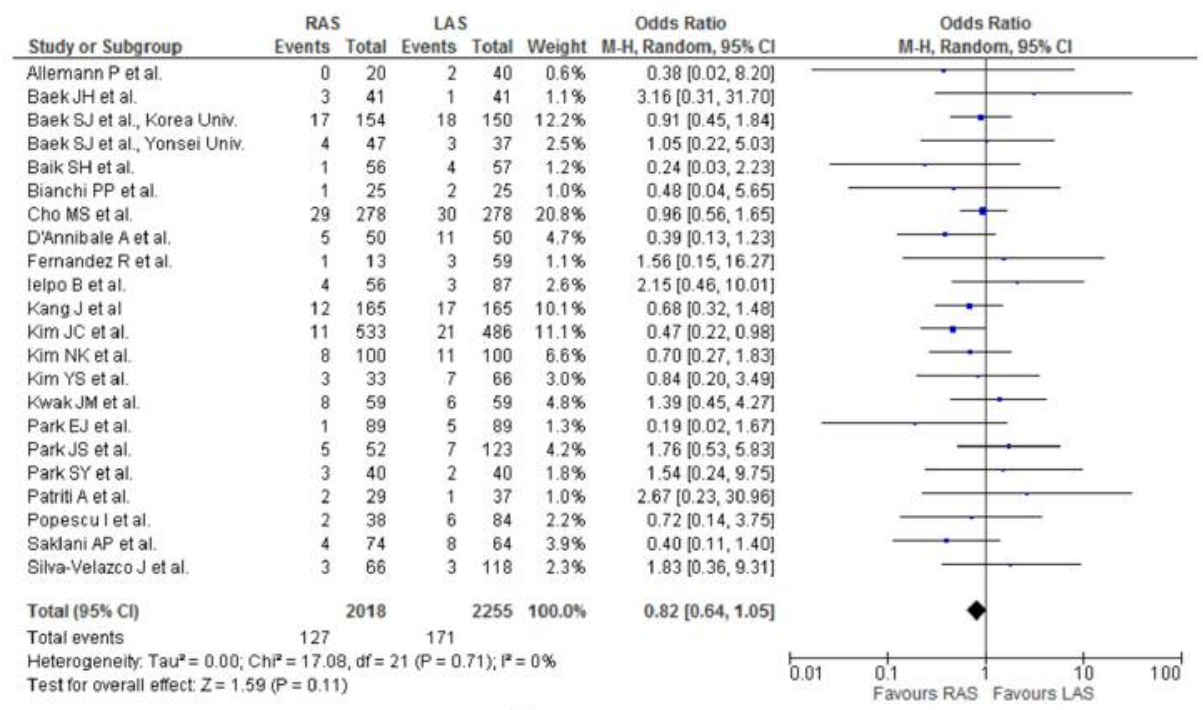

Ileus

RAS LAS Odds Ratio

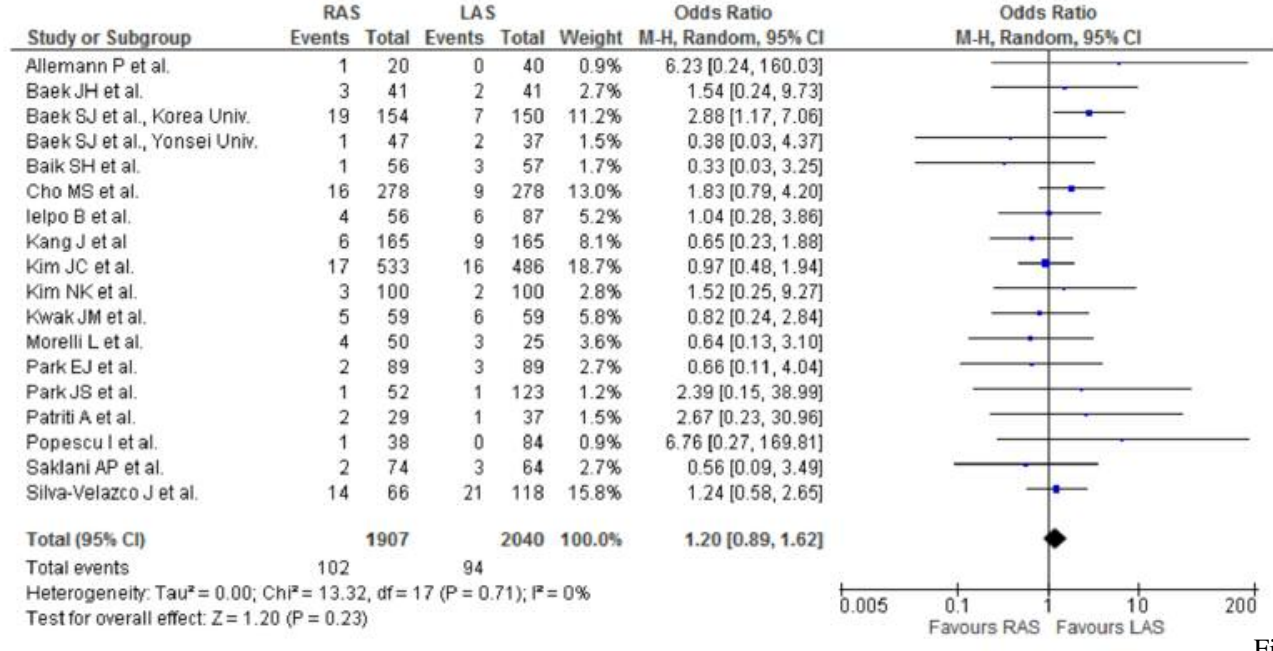

Figure 2. Continued 
C Number of the patients undergoing neoadjuvant chemoradiotherapy (CRT)

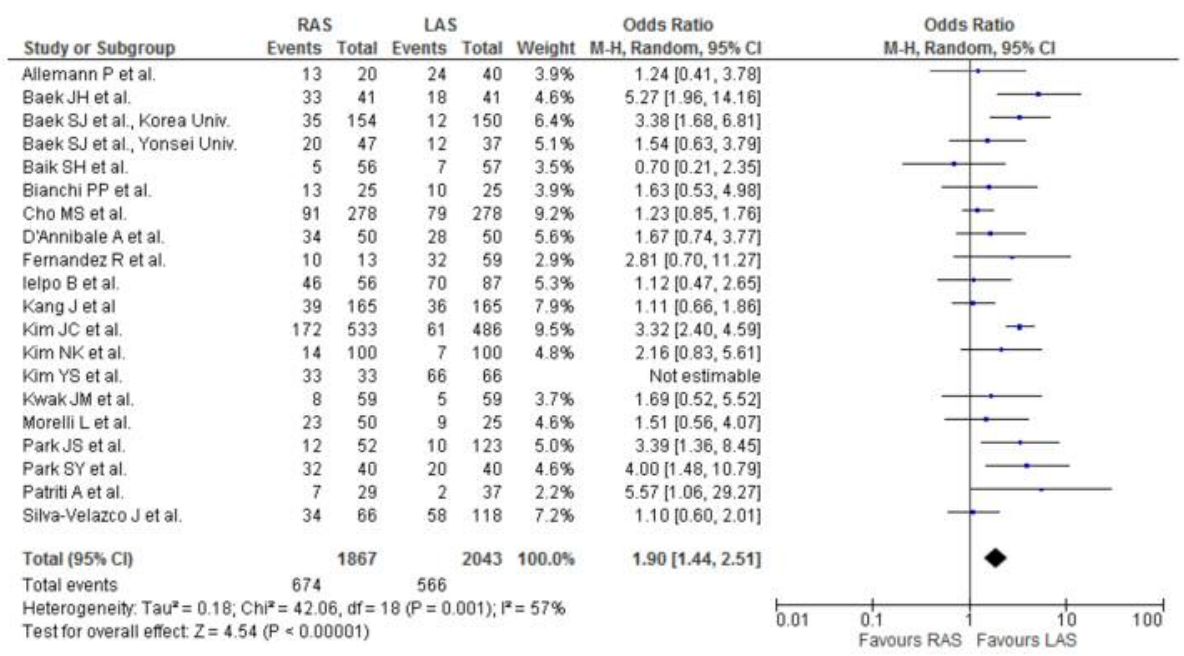

Number of patients with lower rectal cancer

\begin{tabular}{|c|c|c|c|c|c|c|c|c|c|c|}
\hline \multirow[b]{2}{*}{ Study or Subgroup } & \multicolumn{2}{|c|}{ RAS } & \multicolumn{2}{|c|}{ LAS } & \multicolumn{3}{|c|}{ Odds Ratio } & \multirow{2}{*}{\multicolumn{2}{|c|}{$\begin{array}{l}\text { Odds Ratio } \\
\text { M-H, Random, } 95 \% \mathrm{Cl}\end{array}$}} & \\
\hline & Events & Total & Events & Total & Weight & M-H, Random, $95 \% \mathrm{Cl}$ & & & & \\
\hline BaekJH et al. & 15 & 41 & 10 & 41 & $8.3 \%$ & $1.79[0.69,4.65]$ & & & & \\
\hline Cho MS et al. & 69 & 278 & 51 & 278 & $12.1 \%$ & $1.47[0.98,2.21]$ & & & $\leftarrow$ & \\
\hline D'Annibale $A$ et al. & 33 & 50 & 17 & 50 & $9.1 \%$ & $3.77[1.65,8.62]$ & & & $\longrightarrow$ & \\
\hline Fernandez $R$ et al. & 7 & 13 & 11 & 59 & $6.3 \%$ & $5.09[1.43,18.17]$ & & & & \\
\hline lelpo B et al. & 18 & 56 & 27 & 87 & $9.9 \%$ & $1.05[0.51,2.17]$ & & & - & \\
\hline Kang $J$ et al & 57 & 165 & 54 & 165 & $11.8 \%$ & $1.08[0.69,1.71]$ & & & - & \\
\hline Kim JC et al. & 258 & 533 & 86 & 486 & $12.7 \%$ & $4.36[3.27,5.82]$ & & & $\rightarrow$ & \\
\hline Kim NK et al. & 32 & 100 & 19 & 100 & $10.4 \%$ & $2.01[1.04,3.85]$ & & & $\bullet$ & \\
\hline Kwak JM et al. & 24 & 59 & 24 & 59 & $9.8 \%$ & $1.00[0.48,2.08]$ & & & 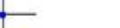 & \\
\hline Park EJ et al. & 18 & 89 & 14 & 89 & $9.5 \%$ & $1.36[0.63,2.93]$ & & & & \\
\hline Total $(95 \% \mathrm{Cl})$ & & 1384 & & 1414 & $100.0 \%$ & $1.86[1.20,2.90]$ & & & & \\
\hline Total events & 531 & & 313 & & & & & & & \\
\hline $\begin{array}{l}\text { Heterogeneity: Tau² } \\
\text { Test for overall effect }\end{array}$ & $\begin{array}{l}0.37 ; \mathrm{Ch} \\
\mathrm{Z}=2.77\end{array}$ & $\begin{array}{l}2=48 . \\
P=0.0\end{array}$ & $\begin{array}{l}\text { 12, } d f=9 \\
06)\end{array}$ & $(P<0.0$ & 00001); & $=81 \%$ & 0.01 & Favours RAS & $\begin{array}{rr}10 \\
\text { Favours LAS }\end{array}$ & 100 \\
\hline
\end{tabular}

Length from anal verge to the lower margin of tumor $(\mathrm{cm})$

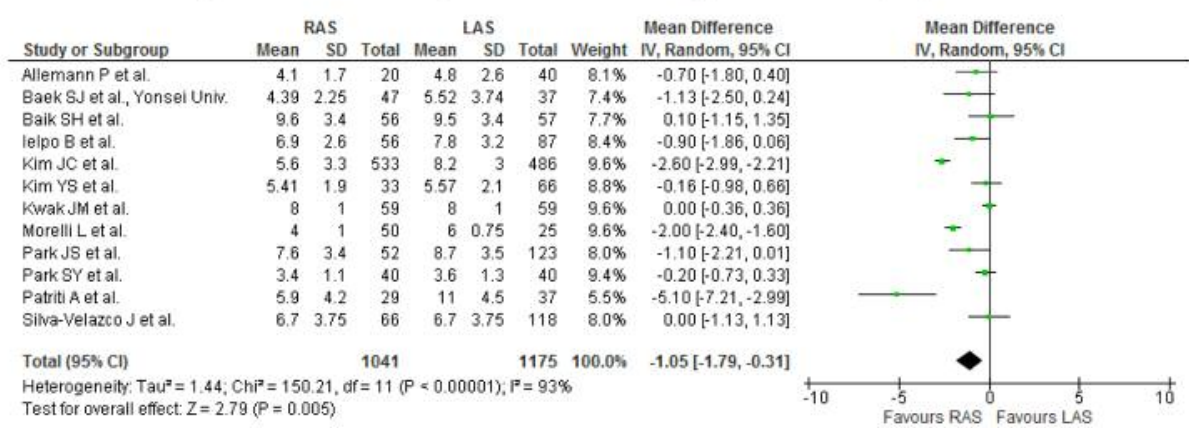

Figure 2. Continued

conducted a meta-analysis, by 12 randomized controlled trials, to evaluate and compare the short- and long-term outcomes of LAS and OS for the treatment of patients with rectal cancer (16). The operative time for OS was significantly shorter, by $40.96 \mathrm{~min}$, than that for LAS. The intraoperative blood loss in OS were significantly more than that in LAS. There were no significant differences in time to bowel movement, time to oral diet and duration of hospital stay. Significant heterogeneity was found in all of them. Differences in the clinical approach at different institutions may have caused the heterogeneity in time to bowel movement, time to oral diet and the duration of hospital stay. 


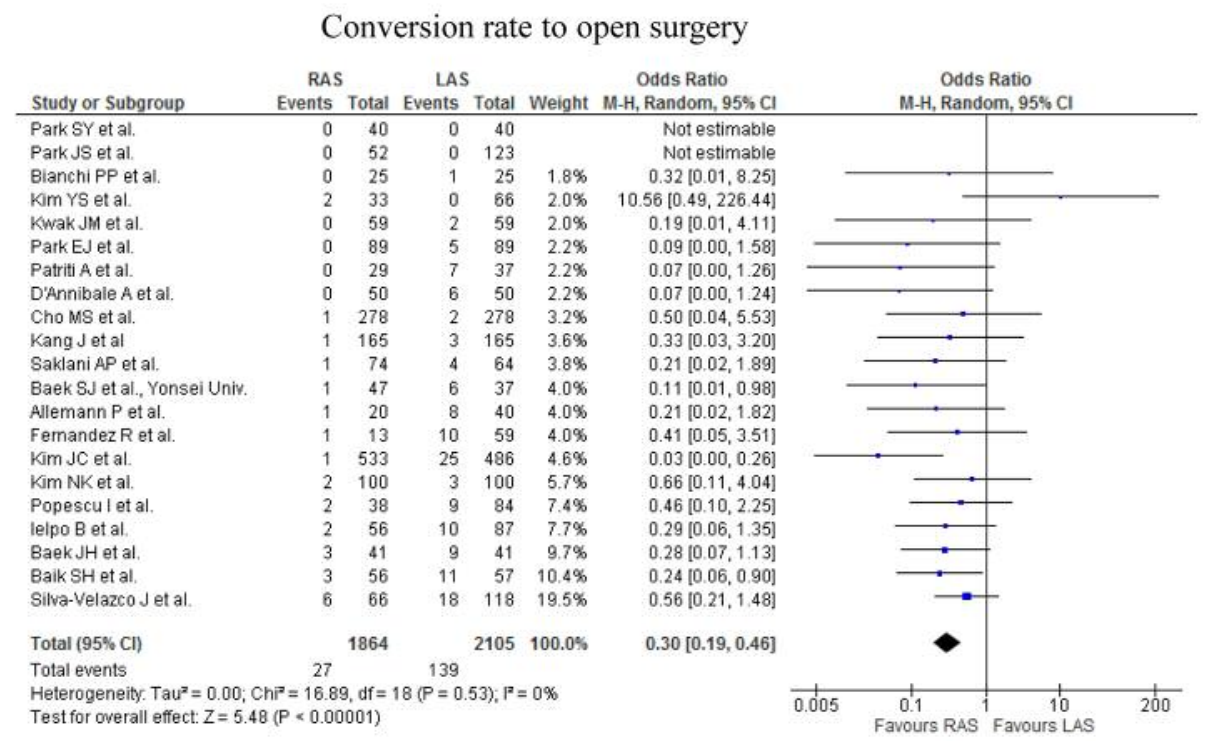

Figure 2. Meta-analysis of the short-term outcomes for rectal cancer. a) The operative time for RAS was significantly greater than that for LAS $(p<0.00001$; upper part). No significant difference was found in the intraoperative estimated blood loss ( $p=0.44$; lower part). $b)$ The occurrence rate of overall complications did not differ significantly between RAS and LAS (upper part). The occurrence rate of anastomotic leakage did not differ significantly between the two procedures (middle part). The occurence rate of ileus did not differ significantly between the two procedures (lower part).c) The rate of the patients undergoing the neoadjuvant chemoradiotherapy was significantly higher in RAS than in LAS (first part). The rate of the patients with lower rectal cancer was significantly higher in RAS than in LAS (second part). The distance from anal verge to the lower margin of tumor was significantly shorter in RAS than in LAS (third part). The conversion rate of RAS was significantly lower than that of LAS (fourth part).

There was no significant difference in overall postoperative complications or in specific complications, such as anastomotic leakage, interperitoneal abscess, wound infection, ileus, postoperative bleeding or DVT between RAS and LAS. There were no cases and three cases of perioperative mortality in RAS and in LAS, respectively. Two cases in LAS were died because of cardiovascular complications, and cause of death of the remaining 1 case is unknown.

In resected specimen, no significant difference was found in number of retrieved lymph nodes, length of CRM, DM and PM, number of cases with inadequate CRM and DM, and number of patients with incomplete TME, between RAS and LAS. The quality of RAS may be almost similar to that of LAS. All of the above-mentioned observations suggest that the safety and feasibility of RAS for rectal cancer is not inferior to that of LAS.

In the long-term period, we found that the rates of overall recurrence, metastatic recurrence and local recurrence are not significantly different between RAS and LAS. We found no significant difference in 3-year overall survival and 3-year disease free survival. These findings suggest that RAS is comparable to LAS with respect to long-term oncologic results and, therefore, may be an optional surgical treatment for rectal cancer.

Eight and 4 articles reported data on urinary and sexual dysfunction, respectively.
In the long-term quality of life, urinary dysfunction did not differ significantly between the 2 groups (Odds Ratio $=0.74 ; 95 \% \mathrm{CI}=0.45-1.20 ; p=0.22)$. There was no significant difference in male erectile dysfunction between RAS and LAS groups. In the previous literature, no significant difference of urinary and sexual dysfunction was found between LAS and OS (6).

There are several limitations in this study. First, all of the 23 articles are non-randomized clinical trials, which may bias to estimating the results. Second, there is a problem regarding the variation of surgical procedures. Yet, most procedures were anterior resection of the rectum, the type of operation - e.g., intersphincteric resection or abdominal peritoneal resection - varied depending on tumor location or condition. Third, twenty of the 23 articles reported the preoperative chemoradiation for rectal cancer. The influence of preoperative chemoradiation to selection for the surgical procedures or prognosis could not be discussed. Fourth, as RAS is a relatively recent procedure, duration of following up patients is not adequate. 3-year over survival and diseasefree interval were reported and estimated in this study. Data for 5-year follow-up may be requested.

In conclusion, although there are several limitations, this meta-analysis showed that RAS for rectal cancer result in almost similar outcomes in the short-term except for a longer operation and a lower conversion rate, but is not inferior in 
Table II. The revised and validated version of MINORS.

\begin{tabular}{|c|c|c|c|c|c|c|c|c|c|c|c|c|c|}
\hline & \multicolumn{9}{|c|}{$\begin{array}{l}\text { Methodological items for } \\
\text { non-randomized studies }\end{array}$} & \multicolumn{4}{|c|}{$\begin{array}{l}\text { Additional criteria in the case } \\
\text { of comparative study }\end{array}$} \\
\hline & $\begin{array}{c}\text { A } \\
\text { clearly } \\
\text { stated } \\
\text { aim }\end{array}$ & $\begin{array}{c}\text { Inclusion } \\
\text { of } \\
\text { con- } \\
\text { secutive } \\
\text { patients }\end{array}$ & $\begin{array}{c}\text { Pro- } \\
\text { spective } \\
\text { collection } \\
\text { of } \\
\text { data }\end{array}$ & $\begin{array}{l}\text { Endpoints } \\
\text { appropriate } \\
\text { to the aim } \\
\text { of the } \\
\text { study }\end{array}$ & $\begin{array}{l}\text { Unbiased } \\
\text { assessment } \\
\text { of } \\
\text { the study } \\
\text { endpoint }\end{array}$ & $\begin{array}{l}\text { Follow-up } \\
\text { period } \\
\text { appropriate } \\
\text { to the aim } \\
\text { of the study }\end{array}$ & $\begin{array}{l}\text { Loss to } \\
\text { follow-up } \\
\text { less } \\
\text { than } \\
5 \%\end{array}$ & $\begin{array}{c}\text { Pro- } \\
\text { spective } \\
\text { calculation } \\
\text { of the } \\
\text { study size }\end{array}$ & $\begin{array}{c}\text { An } \\
\text { adequate } \\
\text { control } \\
\text { group }\end{array}$ & $\begin{array}{l}\text { Contempo- } \\
\text { rary } \\
\text { groups }\end{array}$ & $\begin{array}{l}\text { Baseline } \\
\text { equi- } \\
\text { valence } \\
\text { of } \\
\text { groups }\end{array}$ & $\begin{array}{l}\text { Adequate } \\
\text { statistical } \\
\text { analyses }\end{array}$ & Score \\
\hline $\begin{array}{l}1 \text { Bianchi PP } \\
\text { et al. }\end{array}$ & 2 & 2 & 1 & 2 & 0 & 1 & 2 & 0 & 2 & 2 & 2 & 2 & 18 \\
\hline $\begin{array}{l}2 \text { Popescu } \\
\text { et al. }\end{array}$ & 2 & 2 & 0 & 2 & 0 & 1 & 2 & 0 & 2 & 2 & 2 & 2 & 17 \\
\hline $\begin{array}{l}3 \text { Park JS } \\
\text { et al. }\end{array}$ & 2 & 2 & 1 & 2 & 0 & 0 & 2 & 0 & 2 & 2 & 2 & 2 & 17 \\
\hline $\begin{array}{l}4 \text { Kwak JM } \\
\text { et al. }\end{array}$ & 2 & 2 & 1 & 2 & 0 & 1 & 2 & 0 & 2 & 2 & 2 & 2 & 18 \\
\hline $\begin{array}{l}5 \text { Baek SJ } \\
\text { et al. }\end{array}$ & 2 & 2 & 0 & 2 & 0 & 0 & 2 & 0 & 2 & 2 & 2 & 2 & 16 \\
\hline $\begin{array}{l}6 \text { Saklani AP } \\
\text { et al. }\end{array}$ & 2 & 2 & 1 & 2 & 0 & 1 & 2 & 0 & 2 & 2 & 2 & 2 & 18 \\
\hline $\begin{array}{l}7 \text { Baek SJ } \\
\text { et al. }\end{array}$ & 2 & 2 & 0 & 2 & 0 & 1 & 2 & 0 & 2 & 2 & 2 & 2 & 17 \\
\hline $\begin{array}{l}8 \text { Kang J } \\
\text { et al. }\end{array}$ & 2 & 2 & 1 & 2 & $\begin{array}{c}1 \\
* * *\end{array}$ & 1 & 2 & 0 & 2 & 2 & 2 & 2 & 19 \\
\hline $\begin{array}{l}9 \text { Fernandez } \\
\text { et al. }\end{array}$ & 2 & 2 & 0 & 2 & 0 & 0 & 2 & 0 & 2 & 2 & 2 & 2 & 16 \\
\hline $\begin{array}{l}10 \text { D'Annibale } \\
\text { A et al. }\end{array}$ & 2 & 2 & 1 & 2 & 0 & 0 & 2 & 0 & 2 & 2 & 2 & 2 & 17 \\
\hline $\begin{array}{l}11 \text { Park SY } \\
\text { et al. }\end{array}$ & 2 & 2 & 0 & 2 & 0 & 0 & 2 & 0 & 2 & 2 & 2 & 2 & 16 \\
\hline $\begin{array}{l}12 \mathrm{Kim} \text { YS } \\
\text { et al. }\end{array}$ & 2 & 2 & 1 & 2 & 0 & 0 & 2 & 0 & 2 & 2 & 2 & 2 & 17 \\
\hline $\begin{array}{l}13 \text { Cho MS } \\
\text { et al. }\end{array}$ & 2 & 2 & 0 & 2 & $\begin{array}{c}1 \\
* * *\end{array}$ & 2 & 2 & 0 & 2 & 2 & 2 & 2 & 19 \\
\hline $\begin{array}{l}14 \text { Baik SH } \\
\text { et al. }\end{array}$ & 2 & 2 & 2 & 2 & 1 & 1 & 2 & 1 & 2 & 2 & 2 & 2 & 21 \\
\hline $\begin{array}{l}15 \text { Patriti A } \\
\text { et al. }\end{array}$ & 2 & 2 & 2 & 2 & 1 & 1 & 2 & 1 & 2 & 2 & 2 & 2 & 21 \\
\hline $\begin{array}{l}16 \mathrm{Kim} \mathrm{NK} \\
\text { et al. }\end{array}$ & 2 & 2 & 0 & 2 & 0 & 0 & 2 & 0 & 2 & 2 & 2 & 2 & 16 \\
\hline $\begin{array}{l}17 \text { Baek JH } \\
\text { et al. }\end{array}$ & 2 & 2 & 1 & 2 & 0 & 0 & 2 & 0 & 2 & 2 & 2 & 2 & 17 \\
\hline $\begin{array}{l}18 \text { Ielpo B } \\
\text { et al. }\end{array}$ & 2 & 2 & 0 & 2 & 0 & 0 & 2 & 0 & 2 & 2 & 2 & 2 & 16 \\
\hline $\begin{array}{l}19 \mathrm{Kim} \mathrm{JC} \\
\text { et al. }\end{array}$ & 2 & 2 & 0 & 2 & 0 & 1 & 2 & 0 & 2 & 2 & 2 & 2 & 18 \\
\hline $\begin{array}{l}20 \text { Park EJ } \\
\text { et al. }\end{array}$ & 2 & 2 & 0 & 2 & 0 & 0 & 2 & 0 & 2 & 2 & 2 & 2 & 16 \\
\hline $\begin{array}{l}21 \text { Morelli L } \\
\text { et al. }\end{array}$ & 2 & 2 & 0 & 2 & 0 & 0 & 2 & 0 & 2 & 2 & 2 & 2 & 16 \\
\hline $\begin{array}{l}22 \text { Silva-Velazco } \\
\text { et al. }\end{array}$ & o 2 & 2 & 0 & 2 & 0 & 0 & 2 & 0 & 2 & 2 & 2 & 2 & 16 \\
\hline $\begin{array}{l}23 \text { Allemann P } \\
\text { et al. }\end{array}$ & 2 & 2 & 1 & 2 & 0 & 1 & 2 & 0 & 2 & 2 & 2 & 2 & 18 \\
\hline
\end{tabular}

The items are scored 0 (not reported), 1 (reported but inadequate) or 2 (reported and adequate). The global ideal score being 16 for non-comparative studies and 24 for comparative studies. ${ }^{* * *}$ Case-matched analysis. 


\begin{tabular}{|c|c|c|c|c|c|c|c|c|c|c|}
\hline \multirow[b]{2}{*}{ Study or Subgroup } & \multicolumn{2}{|c|}{ RAS } & \multicolumn{2}{|c|}{ LAS } & \multirow[b]{2}{*}{ Weight } & \multirow{2}{*}{$\begin{array}{c}\text { Odds Ratio } \\
\text { M-H, Random, } 95 \% \mathrm{Cl}\end{array}$} & \multirow{2}{*}{\multicolumn{3}{|c|}{$\begin{array}{c}\text { Odds Ratio } \\
\text { M-H, Random, } 95 \% \mathrm{Cl}\end{array}$}} & \\
\hline & Events & Total & Events & Total & & & & & & \\
\hline Allemann $P$ et al. & 0 & 20 & 1 & 40 & $0.6 \%$ & $0.64[0.03,16.48]$ & & & & \\
\hline Cho MS et al. & 49 & 278 & 53 & 278 & $33.2 \%$ & $0.91[0.59,1.40]$ & & & & \\
\hline Kwak JM et al. & 3 & 55 & 3 & 54 & $2.3 \%$ & $0.98[0.19,5.09]$ & & & & \\
\hline Patriti $A$ et al. & 0 & 29 & 4 & 37 & $0.7 \%$ & $0.13[0.01,2.44]$ & & & & \\
\hline Popescul et al. & 2 & 38 & 5 & 84 & $2.2 \%$ & $0.88[0.16,4.74]$ & & & & \\
\hline Total events & 162 & & 149 & & & & & & & \\
\hline $\begin{array}{l}\text { Heterogeneity: Tauz } \\
\text { Test for overall effect }\end{array}$ & $\begin{array}{l}0.00 ; \mathrm{Ch} \\
\mathrm{Z}=0.60\end{array}$ & $\begin{array}{l}\mathrm{I}^{2}=3.50 \\
(\mathrm{P}=0.5\end{array}$ & if $d f=5($ & $P=0.6$ & 2); $I^{2}=0 \%$ & & 0.005 & $\begin{array}{c}0.1 \\
\text { Favours RAS }\end{array}$ & $\begin{array}{l}10 \\
11 \\
\text { Favours LA }\end{array}$ & 200 \\
\hline
\end{tabular}

3-year overall survival

\begin{tabular}{|c|c|c|c|c|c|c|c|c|c|}
\hline \multirow[b]{2}{*}{ Study or Subgroup } & \multicolumn{2}{|c|}{ RAS } & \multicolumn{2}{|c|}{ LAS } & \multicolumn{2}{|r|}{ Odds Ratio } & \multirow{2}{*}{\multicolumn{2}{|c|}{$\begin{array}{c}\text { Odds Ratio } \\
\text { M- } \mathrm{H}, \text { Random, } 95 \% \mathrm{Cl}\end{array}$}} & \\
\hline & Events & Total & Events & Total & Weight & M-H, Random, $95 \% \mathrm{Cl}$ & & & \\
\hline Baek SJ et al., Yonsei Univ. & 40 & 47 & 34 & 37 & $10.6 \%$ & $0.50[0.12,2.10]$ & & & \\
\hline Kim JC et al. & 504 & 533 & 459 & 486 & $74.4 \%$ & $1.02[0.60,1.75]$ & & & \\
\hline Saklani AP et al. & 67 & 74 & 59 & 64 & $15.0 \%$ & $0.81[0.24,2.69]$ & & & \\
\hline Total $(95 \% \mathrm{Cl})$ & & 654 & & 587 & $100.0 \%$ & $0.92[0.58,1.46]$ & & & \\
\hline Total events & 611 & & 552 & & & & & & \\
\hline $\begin{array}{l}\text { Heterogeneity: } \operatorname{Tau}^{2}=0.00 ; \\
\text { Test for overall effect } Z=0.3\end{array}$ & $\begin{array}{l}(P=0.71 \\
(P=0.8\end{array}$ & i) $d f=2($ & $(P=0.65$ & $; 1^{2}=0$ & & & 0.05 & $\begin{array}{ccc}0.2 & 1 & 5 \\
\text { Favours RAS } & \text { Favours LAS }\end{array}$ & 20 \\
\hline
\end{tabular}

b Number of cases suffering from urinary dysfunction

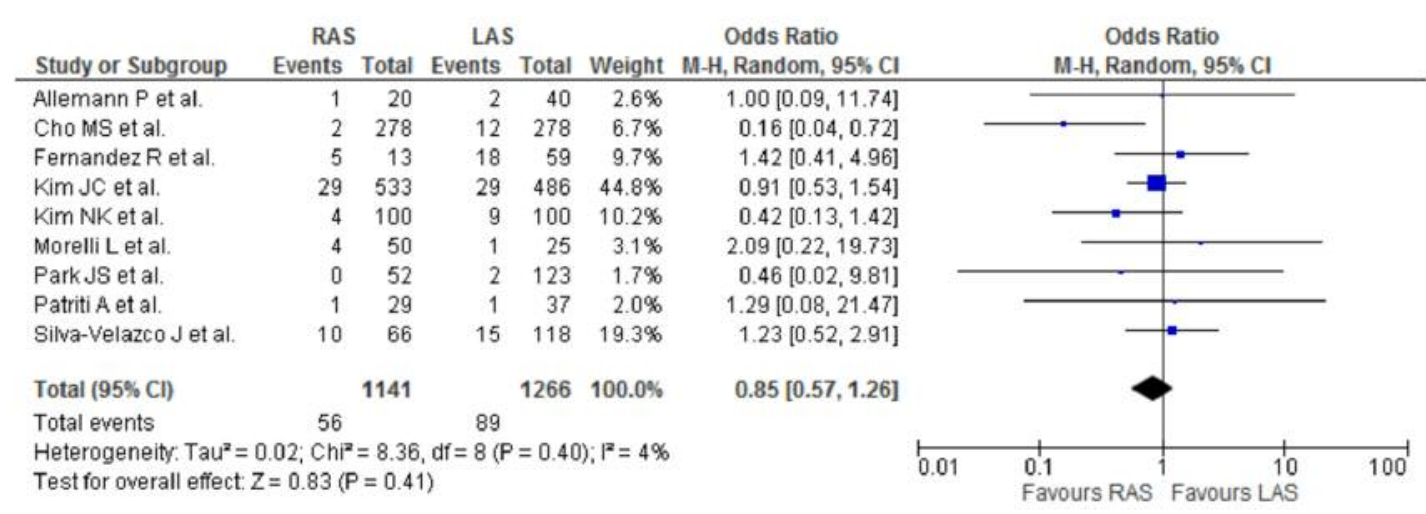

Number of cases suffering from erectile dysfunction

RAS LAS Odds Ratio

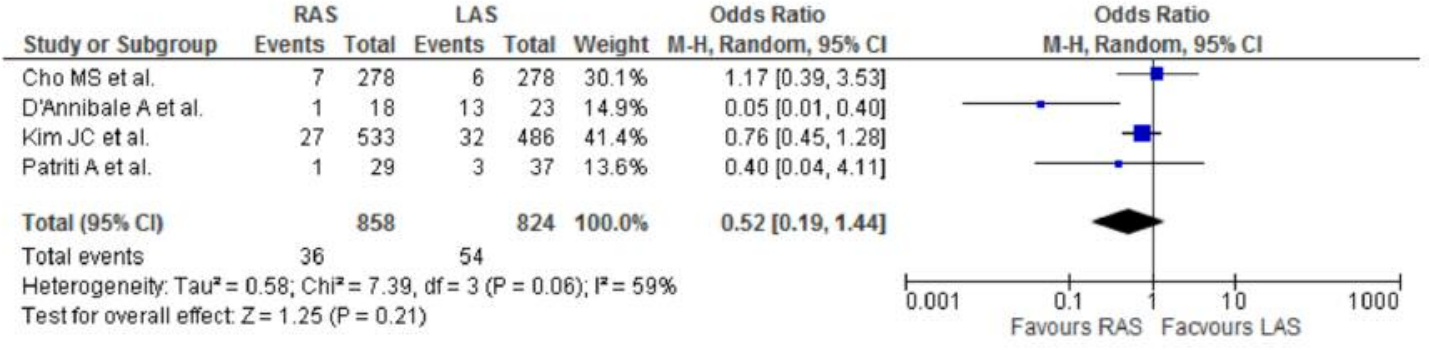

Figure 3. Meta-analysis of long-term outcomes for rectal cancer. a) The rate of overall recurrence was not significantly different between RAS and LAS (upper part). We found no significant difference in 3-year overall survival rate between RAS and LAS (lower part). b) Urinary dysfunction did not differ significantly between the 2 groups (upper part). There was no significant difference in erectile dysfunction between robotic and laparoscopic groups (lower part). 
the long-term oncologic outcomes, compared to LAS. Therefore, RAS may be an acceptable optional treatment to LAS for rectal cancer.

If possible, some prospective randomized trials comparing RAS to LAS for rectal cancer should be performed in the near future.

\section{Conflicts of Interest}

Drs Hiroshi Ohtani, Kiyoshi Maeda, Shinya Nomura, Osamu Shinto, Yoko Mizuyama, Hiroji Nakagawa, Hisashi Nagahara, Masatsune Shibutani, Tatsunari Fukuoka, Ryosuke Amano, Kosei Hirakawa and Masaichi Ohira have no conflicts of interest or financial ties to disclose. All Authors meet the International Committee of Medical Journal Editors authorship criteria.

\section{References}

1 Jacobs M, Verdeja JC and Goldstein HS: Minimally invasive colon resection (laparoscopic colectomy). Surg Laparosc Endosc 1: 144-150, 1991.

2 Clinical Outcomes of Surgical Therapy Study Group, Nelson H, Sargent DJ, Wieand HS, Fleshman J, Anvari M, Stryker SJ, Beart RW Jr, Hellinger M, Flanagan R Jr., Peters W and Ota D: A comparison of laparoscopically assisted and open colectomy for colon cancer. N Engl J Med 350: 2050-2059, 2004.

3 Ohtani H, Tamamori Y, Arimoto Y, Nishiguchi Y, Maeda K and Hirakawa K: A meta-analysis of the short- and long-term results of randomized controlled trials that compared laparoscopyassisted and open colectomy for colon cancer. J Cancer 3: 4957, 2012.

4 Guillou PJ, Quirke P, Thorpe H, Walker J, Jayne DG, Smith AM, Heath RM and Brown JM; MRC CLASICC trial group: Shortterm endpoints of conventional versus laparoscopic-assisted surgery in patients with colorectal cancer (MRC CLASICC trial): multicentre, randomised controlled trial. Lancet 365: 1718-1726, 2005

5 Green BL, Marshall HC, Collinson F, Quirke P, Guillou P, Jayne DG and Brown JM: Long-term follow-up of the Medical Research Council CLASICC trial of conventional versus laparoscopically assisted resection in colorectal cancer. Br J Surg 100: 75-82, 2013.

6 Ohtani H, Tamamori Y, Azuma T, Mori Y, Nishiguchi Y, Maeda $\mathrm{K}$ and Hirakawa $\mathrm{K}$ : A meta-analysis of the short- and long-term results of randomized controlled trials that compared laparoscopy-assisted and conventional open surgery for rectal cancer. J Gastrointest Surg 15: 1375-1385, 2011.

7 Ng SS, Leung KL, Lee JF, Yiu RY, Li JC and Hon SS: Longterm morbidity and oncologic outcomes of laparoscopic-assisted anterior resection for upper rectal cancer: ten-year results of a prospective, randomized trial. Dis Colon Rectum 52: 558-566, 2009.

8 Weber PA, Merola S, Wasielewski A and Ballantyne GH: Telerobotic-assisted laparoscopic right and sigmoid colectomies for benign disease. Dis Colon Rectum 45: 1689-1694, 2002.

9 Slim K, Nini E, Forestier D, Kwiatkowski F, Panis Y and Chipponi J: Methodological index for non-randomized studies (minors): development and validation of a new instrument. ANZ J Surg 73: 712-716, 2003
10 Bianchi PP, Ceriani C, Locatelli A, Spinoglio G, Zampino MG, Sonzogni A, Crosta C and Andreoni B: Robotic versus laparoscopic total mesorectal excision for rectal cancer: a comparative analysis of oncological safety and short-term outcomes. Surg Endosc 24: 2888-2894, 2010.

11 Popescu I, Vasilescu C, Tomulescu V, Vasile S and Sgarbura O: The minimally invasive approach, laparoscopic and robotic, in rectal resection for cancer. A single center experience. Acta Chir Iugos1 57: 29-35, 2010.

12 Park JS, Choi GS, Lim KH, Jang YS and Jun SH: a comparison of robot-assisted, laparoscopic, and open surgery in the treatment of rectal cancer. Surg Endosc 25: 240-248, 2011.

13 Kwak JM, Kim SH, Kim J, Son DN, Baek SJ, Cho JS Kwak JM, Kim SH, Kim J, Son DN, Baek SJ and Cho JS: Robotic vs laparoscopic resection of rectal cancer: short-term outcomes of a case-control study. Dis Colon Rectum 54: 151-156, 2011.

14 Baek SJ, Kim SH, Cho JS, Shin JW and Kim J: Robotic versus conventional laparoscopic surgery for rectal cancer: a cost analysis from a single institute in Korea. World J Surg 36: 2722-2729, 2012.

15 Saklani AP, Lim DR, Hur H, Min BS, Baik SH, Lee KY and Kim NK: Robotic versus laparoscopic surgery for mid-low rectal cancer after neoadjuvant chemoradiation therapy: comparison of oncologic outcomes. Int J Colorectal Dis 28: 1689-1698, 2013.

16 Baek SJ, Al-Asari S, Jeong DH, Hur H, Min BS, Baik SH and Kim NK: Robotic versus laparoscopic coloanal anastomosis with or without intersphincteric resection for rectal cancer. Surg Endosc 27: 4157-4163, 2013.

17 Kang J, Yoon KJ, Min BS, Hur H, Baik SH, Kim NK and Lee KY: The impact of robotic surgery for mid and low rectal cancer: a case-matched analysis of a 3-arm comparison--open, laparoscopic, and robotic surgery. Ann Surg 257: 95-101, 2013.

18 Fernandez R, Anaya DA, Li LT, Orcutt ST, Balentine CJ, Awad SA, Berger DH, Albo DA and Artinyan A: Laparoscopic versus robotic rectal resection for rectal cancer in a veteran population. Am J Surg 206: 509-517, 2013.

19 D'Annibale A, Pernazza G, Monsellato I, Pende V, Lucandri G, Mazzocchi P and Alfano G: Total mesorectal excision: a comparison of oncological and functional outcomes between robotic and laparoscopic surgery for rectal cancer. Surg Endosc 27: 1887-1895, 2013.

20 Park SY, Choi GS, Park JS, Kim HJ and Ryuk JP: Short-term clinical outcome of robot-assisted intersphincteric resection for low rectal cancer: a retrospective comparison with conventional laparoscopy. Surg Endosc 27: 48-55, 2013.

21 Kim YS, Kim MJ, Park SC, Sohn DK, Kim DY, Chang HJ, Nam $\mathrm{BH}$ and $\mathrm{Oh} \mathrm{JH}$ : Robotic versus laparoscopic surgery for rectal cancer after preoperative chemoradiotherapy: Case-matched study of short-term outcomes. Cancer Res Treat 48: 225-231, 2016.

22 Cho MS, Baek SJ, Hur H, Min BS, Baik SH, Lee KY and Kim NK: Short and long-term outcomes of robotic versus laparoscopic total mesorectal excision for rectal cancer: a casematched retrospective study. Medicine 94: e522, 2015.

23 Baik SH, Kwon HY, Kim JS, Hur H, Sohn SK, Cho CH and Kim H: Robotic versus laparoscopic low anterior resection of rectal cancer: short-term outcome of a prospective comparative study. Ann Surg Oncol 16: 1480-1487, 2009.

24 Patriti A, Ceccarelli G, Bartoli A, Spaziani A, Biancafarina A and Casciola L: Short- and medium-term outcome of robotassisted and traditional laparoscopic rectal resection. JSLS 13: 176-183, 2009. 
25 Kim NK and Kang J: Optimal Total Mesorectal Excision for Rectal Cancer: the Role of Robotic Surgery from an Expert's View. J Korean Soc Coloproctol 26: 377-387, 2010.

26 Baek JH, Pastor C and Pigazzi A: Robotic and laparoscopic total mesorectal excision for rectal cancer: a case-matched study. Surg Endosc 25: 521-525, 2011

27 Ielpo B, Caruso R, Quijano Y, Duran H, Diaz E, Fabra I, Oliva C, Olivares S, Ferri V, Ceron R, Plaza C and Vicente E: Robotic versus laparoscopic rectal resection: is there any real difference? A comparative single center study. Int J Med Robot 10: 300-305, 2014.

28 Kim JC, Yu CS, Lim SB, Park IJ, Kim CW and Yoon YS: Comparative analysis focusing on surgical and early oncological outcomes of open, laparoscopy-assisted, and robot-assisted approaches in rectal cancer patients. Int J Colorectal Dis 31: 1179-1187, 2016.

29 Park EJ, Kim CW, Cho MS, Kim DW, Min BS, Baik SH, Lee $\mathrm{KY}$ and Kim NK: Is the learning curve of robotic low anterior resection shorter than laparoscopic low anterior resection for rectal cancer?: a comparative analysis of clinicopathologic outcomes between robotic and laparoscopic surgeries. Medicine 93: e109, 2014.

30 Morelli L, Guadagni S, Lorenzoni V, Di Franco G, Cobuccio L, Palmeri M, Caprili G, D'Isidoro C, Moglia A, Ferrari V, Di Candio G, Mosca F and Turchetti G: Robot-assisted versus laparoscopic rectal resection for cancer in a single surgeon's experience: a cost analysis covering the initial 50 robotic cases with the da Vinci Si. Int J Colorectal Dis 31: 1639-1648, 2016.
31 Silva-Velazco J, Dietz DW, Stocchi L, Costedio M, Gorgun E, Kalady MF, Kessler H, Lavery IC and Remzi FH: considering value in rectal cancer surgery: an analysis of costs and outcomes based on the open, laparoscopic, and robotic approach for proctectomy. Ann Surg 265: 960-968, 2017.

32 Allemann P, Duvoisin C, Di Mare L, Hübner M, Demartines N and Hahnloser D: Robotic-assisted surgery improves the quality of total mesorectal excision for rectal cancer compared to laparoscopy: Results of a Case-Controlled Analysis. World J Surg 40: 1010-1016, 2016.

33 Liberati A, Altman DG, Tetzlaff J, Mulrow C, Gøtzsche PC, Ioannidis JP, Clarke M, Devereaux PJ, Kleijnen J and Moher D: The PRISMA statement for reporting systematic reviews and meta-analyses of studies that evaluate health care interventions: explanation and elaboration. J Clin Epidemiol 62: e1-34, 2009.

34 Hozo SP, Djulbegovic B and Hozo I: Estimating the mean and variance from the median, range, and the size of a sample. BMC Med Res Methodol 20: 5-13, 2005.

35 Choi GS, Park IJ, Kang BM, Lim KH and Jun SH: A novel approach of robotic-assisted anterior resection with transanal or transvaginal retrieval of the specimen for colorectal cancer. Surg Endosc 23: 2831-2835, 2005.
Received January 14, 2018

Revised February 12, 2018

Accepted February 20, 2018 